\title{
QF-3 RINGS AND TORSION THEORIES
}

\author{
JOSÉ L. GÓMEZ PARDO and NIEVES RODRÍGUEZ GONZALEZ
}

(Received 3 February 1987)

Communicated by B. J. Gardner

\begin{abstract}
In this paper, the rings which have a torsion theory $\tau$ with associated torsion radical $t$ such that $R / t(R)$ has a minimal $\tau$-torsionfree cogenerator are studied. When $\tau$ is the trivial torsion theory these are precisely the left QF-3 rings. For $\tau=\tau_{L}$, the Lambek torsion theory, this class of rings is wider but, with an additional hypothesis on $\tau_{L}$ it is shown that if $R$ has this property with respect to the Lambek torsion theory on both sides, then $R$ is a (left and right) QF-3 ring. The results obtained are applied to get new characterizations of QF-3 rings with the ascending chain condition on left annihilators.
\end{abstract}

1980 Mathematics subject classification (Amer. Math. Soc.) (1985 Revision): 16 A 36, 16 A 63.

A ring $R$ is called left QF-3 if it has a minimal faithful left $R$-module and left QF- $3^{\prime}$ if the injective envelope $E\left({ }_{R} R\right)$ is a torsionless module. These rings have been the object of extensive study (for example in $[3,9,11,12,13,16]$ ) and, recently, Baccella has obtained in [2] structure results for the class of nonsingular, finite-dimensional QF-3 rings. In the present paper we consider a (hereditary) torsion theory $\tau$ in $R$-mod with associated torsion radical $\mathbf{t}$ and the property that $R / \mathbf{t}(R)$ has a minimal $\tau$-torsionfree cogenerator $X$, in the sense that $X$ is a $\tau$-torsionfree module which cogenerates $R / \mathrm{t}(R)$ and is a direct summand of every $\tau$-torsionfree cogenerator of $R / \mathbf{t}(R)$. When $\tau$ is the trivial torsion theory in which all $R$-modules are $\tau$-torsionfree, this property defines left $\mathrm{QF}-3$ rings. The class of rings which have this property with respect to the Lambek torsion theory $\tau_{L}$ is wider than the class of left QF-3 rings but when $\tau_{L}$ is strongly semiprime and $R$ has this property for the Lambek torsion theory on both sides,

This work was partially supported by the CAICYT (0784-84).

(C) 1989 Australian Mathematical Society 0263-6115/89 \$A2.00 + 0.00 
then we show that $R$ is a (left and right) QF-3 ring (Theorem 4). This allows us to get characterizations of the class of QF-3 rings with the ascending chain condition (ACC) on left annihilators, which is shown to be equal to the class of $\mathrm{QF}-3^{\prime}$ rings with $\mathrm{ACC}$ on left annihilators.

Our property behaves nicely when $R$ is, furthermore, supposed to be $\tau$-artinian and this case is studied in Theorem 7. For trivial $\tau$ the conditions of this theorem reduce to known characterizations of left artinian left QF-3 rings (in particular, we get as a consequence the main result of [12]) but, taking $\tau$ equal to the Lambek torsion theory we obtain new characterizations of left $\mathrm{QF}-3^{\prime}$ rings with the descending chain condition on left annihilators, QF-3 rings with ACC on left annihilators and, in the nonsingular case, of finite-dimensional left $\mathrm{QF}-3^{\prime}$ rings.

Throughout this paper, $R$ denotes an associative ring with identity and $R$ $\bmod (\bmod -R)$ the category of left (right) $R$-modules. A module $N$ is said to be a cogenerator of $M$ if there is a monomorphism from $M$ to a direct product $N^{I}$ of copies of $N$; in this case we also say that $M$ is $N$-torsionless and, in particular, the ${ }_{R} R$-torsionless modules are called simply torsionless modules. We use the notation $E(M)$ to stand for an injective envelope of the module $M$. When $R$ is left and right QF-3, we will say simply that $R$ is a QF-3 ring and the analogous convention holds for other classes of rings (for example QF-3' rings).

If $\tau$ is a torsion theory of $R$-mod, a submodule $N$ of $M$ is said to be $\tau$-dense in $M$ if $M / N$ is a $\tau$-torsion module; $M$ is $\tau$-finitely generated when it has a finitely generated $\tau$-dense submodule; and $M$ is called $\tau$-noetherian ( $\tau$-artinian) if the lattice $\mathrm{C}_{\tau}(M)$ of $\tau$-closed submodules of $M$ (that is, of those submodules $X$ of $M$ such that $M / X$ is $\tau$-torsionfree) satisfies the ascending chain condition (ACC for short), respectively the descending chain condition (DCC). The Teply-Miller theorem [10, Theorem 1.4] asserts that if $R$ is a $\tau$-artinian ring (that is, ${ }_{R} R$ is $\tau$-artinian), then every $\tau$-artinian module is $\tau$-noetherian. Also, it is well known that if $M$ is $\tau$-noetherian, then $M$ is $\tau$-finitely generated. The Lambek torsion theory of $R-\bmod \tau_{L}$ is the torsion theory cogenerated by $E\left({ }_{R} R\right)$ [15], that is, the $\tau_{L}$-torsionfree modules are precisely the $E\left({ }_{R} R\right)$-torsionless modules. The $\tau_{L}$-finitely generated modules are also called almost finitely generated modules and the elements of $C_{\tau_{L}}(R)$ are called rationally closed left ideals.

The reader is referred to [1], [7] and [15] for all ring-theoretic and torsiontheoretic notions used in the text.

Recall that a torsion theory $\tau$ is called jansian if the corresponding torsion class is closed under direct products or, equivalently, $R$ has a smallest $\tau$-dense left ideal. Then we have

PROPOSITION 1. Let $\tau$ be a torsion theory of $R$-mod. If $R / \mathrm{t}(R)$ is a left $\mathrm{QF}-3$ ring, then $R / \mathrm{t}(R)$ has a minimal $\tau$-torsionfree cogenerator. If, furthermore, $\tau$ is jansian, then the converse holds. 
Proof. Assume first that $R / \mathrm{t}(R)$ is left QF-3 and let $X$ be a minimal faithful left $R / \mathrm{t}(R)$-module. Then $X$ is isomorphic to a left ideal of $R / \mathrm{t}(R)$ and hence it is $\tau$-torsion free as left $R$-module. If $Y$ is a $\tau$-torsion free $R$-module which cogenerates $R / \mathrm{t}(R)$, then $Y$ is canonically a faithful left $R / \mathrm{t}(R)$-module and hence $X$ is a direct summand of $Y$ (as $R / t(R)$-module and also as $R$-module), which shows that it is a minimal $\tau$-torsionfree cogenerator.

Conversely, assume that $\tau$ is jansian and let $X$ be a minimal $\tau$-torsionfree cogenerator of $R / \mathrm{t}(R)$. Then $X$ is a faithful $R / \mathrm{t}(R)$-module and if $Y$ is another faithful $R / \mathrm{t}(R)$-module, it follows from the facts that $R / \mathrm{t}(R)$ is $\tau$-torsionfree and that $\mathrm{t}$ preserves products that $Y / \mathrm{t}(Y)$ is also a cogenerator of $R / \mathrm{t}(R)$. Then our hypothesis implies that $X$ is a direct summand of $Y / t(Y)$. On the other hand, $X$ is a direct summand of $R / \mathbf{t}(R)$ and hence it is $R / \mathbf{t}(R)$-projective, from which it follows easily that $X$ is also a direct summand of $Y$, which completes the proof.

The ring considered in the remarks following Theorem 4, with the Lambek torsion theory $\tau_{L}$, shows that the converse of Proposition 1 is not true in general. On the other hand, if $R$ is a right perfect ring, then every torsion theory of $R$ mod is jansian [15, Corollary VIII.6.3]. In particular $\tau_{L}$ is jansian and so $R$ is left QF-3 if and only if it has a minimal $\tau_{L}$-torsionfree faithful module.

Let $\tau$ be a torsion theory and denote by $M_{\tau}$ the module of quotients corresponding to $M$ (so that $R_{\tau}$ is the ring of quotients of $R$ with respect to $\tau$ ). Then we recall that $\tau$ is called perfect when the localization functor $R$-mod $\rightarrow R_{\tau}$ mod which assigns to each $M$ its module of quotients $M_{\tau}$ is naturally equivalent to the functor $R_{\tau} \otimes_{R^{-}}: R$-mod $\rightarrow R_{\tau}$-mod. This is equivalent to every $R_{\tau}$ module being $\tau$-torsionfree as $R$-module [7, Proposition 45.1]. We have

PROPOSITION 2. Let $\tau$ be a perfect torsion theory. If $R / \mathrm{t}(R)$ has a minimal $\tau$-torsionfree cogenerator, then $R_{\tau}$ is a left QF-3 ring.

Proof. Let $X$ be a minimal $\tau$-torsionfree cogenerator of $R / t(R)$ and $E$ an injective cogenerator of $\tau$ (so that the $\tau$-torsionfree modules are precisely the $E$-torsionless modules). Then $X$ is a direct summand of $E$ and hence it is injective and $\tau$-torsionfree. Thus $X$ is an injective $R_{\tau}$-module in a canonical way [15, Proposition IX.2.7]. Since $X$ is an injective $R$-module, it is clear that $X$ cogenerates $E(R / \mathbf{t}(R))$ and since $R / \mathrm{t}(R)$ is an essential left $R$-submodule of $R_{\tau}$ and $E(R / \mathrm{t}(R))=E\left({ }_{R_{r}} R_{r}\right)$, we see that $X$ cogenerates $R_{\tau}$. Now, let $Y$ be a faithful $R_{\tau}$-module. As $\tau$ is perfect, $Y$ is a $\tau$-torsionfree $R$-module which cogenerates $R / \mathbf{t}(R)$ and hence $X$ is, by hypothesis, a direct summand of $Y$, which shows that $X$ is a minimal faithful $R_{r}$-module.

The converse of Proposition 2 does not hold. For instance, if $R=\mathbf{Z}$ and $\tau$ is the usual torsion theory, then $\mathbf{Z}$ has not a minimal torsionfree cogenerator. 
We recall that a module is called finitely cogenerated if it has a finitely generated essential socle and, more generally, for a torsion theory $\tau, M$ is $\tau$-finitely cogenerated [4] when $M / t(M)$ contains an essential finite direct sum of $\tau$-cocritical modules (a module $C$ is $\tau$-cocritical when it is $\tau$-torsionfree and each proper quotient of $C$ is a torsion module [7]). In [3] it is shown that $R$ is left QF-3 if and only if it has a finitely cogenerated injective faithful left ideal. In general we cannot get a similar result in our situation, for a torsion theory $\tau$ needs not have any $\tau$-cocritical module. If $\tau$ is cogenerated by the injective envelopes of the $\tau$-cocritical modules, then $\tau$ is called strongly semiprime [7] and in this case we have

PROPOSITION 3. Let $\tau$ be a strongly semiprime torsion theory. Then $R / \mathbf{t}(R)$ has a minimal $\tau$-torsionfree cogenerator if and only if it has an injective cogenerator submodule which is $\tau$-finitely cogenerated.

Proof. Necessity. Let $X$ be a minimal $\tau$-torsionfree cogenerator of $R / \mathbf{t}(R)$ and $E=\bigoplus_{I} E_{i}$, where $\left\{E_{i}\right\}$ is a representative set of the isomorphism classes of injective envelopes $E_{i}=E\left(C_{i}\right)$ of $\tau$-cocritical modules $C_{i}$. Since $\tau$ is strongly semiprime, $E$ cogenerates $R / \mathrm{t}(R)$ and hence $X$ is a direct summand of $E$. But $X$ is also a direct summand of $R / \mathrm{t}(R)$ and hence it is a cyclic left $R$-module, so that $X$ is, in fact, a direct summand of a finite direct sum $\bigoplus_{1}^{n} E_{i}=E\left(\bigoplus_{1}^{n} C_{i}\right)$. Thus $X$ is $\tau$-finitely cogenerated by [4, Proposition 1.6].

Sufficiency. Let $Q$ be an injective cogenerator submodule of $R / \mathrm{t}(R)$ such that $Q$ is $\tau$-finitely cogenerated. Then $Q=\bigoplus_{1}^{n} E_{i}$ where $E_{i}=E\left(C_{i}\right)$ is an injective envelope of a $\tau$-cocritical module $C_{i}$. Let $\left\{E_{j}: j=1, \ldots, r\right\}$ be a set of representatives of the isomorphism classes of these injective envelopes and set $X=\bigoplus_{1}^{r} E_{j}$. Let $Y$ be a $\tau$-torsionfree cogenerator of $R / t(R)$. Then $Y$ cogenerates $X$ and hence it cogenerates each $E_{j}$. Therefore, for $j=1, \ldots, r$, there exists a nonzero homomorphism $f_{j}: E_{j} \rightarrow Y$. The restriction of $f_{j}$ to $C_{j}$ is a monomorphism (for $C_{j}$ is $\tau$-cocritical and $Y \tau$-torsionfree) and since $C_{j}$ is essential in $E_{j}$ we see that $f_{j}$ must also be a monomorphism. On the other hand, if $\operatorname{Im} f_{j} \cap \operatorname{Im} f_{k} \neq 0$ for $j \neq k$, then we would have that, since $E_{j}$ and $E_{k}$ are uniform, $E_{j} \simeq E\left(\operatorname{Im} f_{j} \cap \operatorname{Im} f_{k}\right) \simeq E_{k}$, contradiction. Therefore $Y$ contains a submodule isomorphic to $X$ and the proof is complete.

Let now $\tau_{L}$ be the Lambek torsion theory. Then $R$ is $\tau_{L}$-torsionfree and the $\tau_{L}$-torsionfree modules are the $E\left({ }_{R} R\right)$-torsionless modules, so $R$ is left QF-3 relative to $\tau_{L}$ if and only if it has a minimal $E\left({ }_{R} R\right)$-torsionless faithful $R$-module. For these rings, we have

PROPOSITION 4. Let $R$ be a ring such that $\tau_{L}$ is strongly semiprime and $R$ has a minimal $E\left({ }_{R} R\right)$-torsionless faithful left $R$-module and a minimal $E\left(R_{R}\right)$ torsionless faithful right $R$-module. Then $R$ is a QF-3 ring. 
PrOOF. Let $X$ be a minimal $E\left({ }_{R} R\right)$-torsionless faithful left $R$-module. Then $X$ is, by Proposition 3, a $\tau_{L}$-finitely cogenerated injective left ideal of $R$. By [6, Corollaries 1.6 and 1.9] ${ }_{R} X$ is a dominant module in the sense of Kato (see [14]) so that by [14, Theorem 1.4] the trace of $X$ in $R$ is a minimal dense (that is, Lambek-dense) right ideal. Thus the Lambek torsion theory of mod $-R$ is jansian and hence $R$ is right QF-3 by Proposition 1 . The same argument as before shows that the Lambek torsion theory on the left, $\tau_{L}$, is also jansian and, using again Proposition 1 we get that $R$ is left QF-3.

REMARKS. The one-sided version of Theorem 4 fails; that is, there is a ring such tht $\tau_{L}$ is perfect (and so is the Lambek torsion theory of mod $-R$ ) and $R$ has a minimal $E\left({ }_{R} R\right)$-torsionless faithful left $R$-module but $R$ is neither a left nor a right QF-3 ring. An easy example is given by the triangular matrix ring $R=\left(\begin{array}{l}\mathbf{Z} \\ 0\end{array}\right.$ minimal among the $E\left({ }_{R} R\right)$-torsionless faithful left $R$-modules for if $Y$ is another module with this property, then there is a nonzero homomorphism $f: \operatorname{Re} \rightarrow Y$ and since $Y$ is $E\left({ }_{R} R\right)$-torsionless, there is a homomorphism $g: Y \rightarrow E\left({ }_{R} R\right)$ such that $g \circ f \neq 0$. But it is easily seen that $g \circ f$ must be a monomorphism and hence $f$ is also a monomorphism, so that $R e$ is a direct summand of $Y$. Note also that since the maximal (left and right) ring of quotients of $R$ is the full matrix ring $M_{2}(\mathbf{Q})$, both Lambek torsion theories of $R$ are perfect (and hence strongly semiprime).

The triangular matrix ring considered in the above remark shows that the class of left QF-3' rings with ACC on left annihilators is larger than the class of left QF-3 rings with ACC on left annihilators. This cannot happen in the two-sided case, for we have

COROLLARY 5. Let $R$ be a ring with ACC (or DCC) on left annihilators. Then the following conditions are equivalent:

(i) $R$ is a QF-3 ring;

(ii) $R$ is a QF-3' ring;

(iii) $R$ has a minimal $E\left({ }_{R} R\right)$-torsionless faithful left module and a minimal $E\left(R_{R}\right)$-torsionless faithful right module.

PROOF. Note that, in all cases, every element of $E\left({ }_{R} R\right)\left(E\left(R_{R}\right)\right)$ is also an element of a torsionless module and hence the left (right) annihilator ideals of $R$ coincide with the annihilators of subsets of $E\left({ }_{R} R\right)$ (respectively $E\left(R_{R}\right)$ ). Thus the ACC (DCC) on left annihilators is equivalent to $R$ being $\tau_{L^{-}}$-noetherian $\left(\tau_{L^{-}}\right.$ artinian) and similarly on the right. In particular, $\tau_{L}$ is strongly semiprime (see [7, page 324]) and so the equivalence between (i) and (iii) follows from Theorem 4. Since (i) implies (ii) obviously, to complete the proof it is enough to show that (ii) implies (iii). Since $R$ has ACC on left annihilators, it has DCC on right 
annihilators and hence, by the Teply-Miller theorem [10, Theorem 1.4], it has ACC on right annihilators. Thus $R$ has also DCC on left annihilators, that is, $R$ is $\tau_{L}$-artinian and by [10, Corollary 3.3] $E\left({ }_{R} R\right)$ is a finite direct sum of injective envelopes of $\tau_{L}$-cocritical modules. Taking the direct sum of representatives of each isomorphism class of these injective envelopes we have a module $X$ which is clearly faithful and, since $R$ is left $\mathrm{QF}-3^{\prime}, X$ is in fact isomorphic to a left ideal of $R$. Now, it follows from Proposition 3 that $R$ has a minimal $\tau_{L}$-torsionfree cogenerator, that is, a minimal $E\left({ }_{R} R\right)$-torsionless faithful left module. Since $R$ has also DCC on right annihilators and is right $Q F-3^{\prime}$, the analogous result holds on the right and the proof is complete.

We will make use of the following lemma.

LEMMA 6. Let $R$ be a $\tau$-artinian ring and $C$ a $\tau$-cocritical module such that every $\tau$-finitely generated submodule of $E(C)$ is cogenerated by $R / \mathrm{t}(R)$. Then $E(C)$ is a $\tau$-noetherian module.

ProOF. See the proof that (i) implies (ii) in [8, Theorem 3.1].

Left artinian left QF-3 rings can be characterized by the property that every finitely generated submodule of $E\left({ }_{R} R\right)$ is torsionless (this was proved by Rutter in [12]), and also as the left noetherian rings such that $E\left({ }_{R} R\right)$ is projective. We are going to give a torsion-theoretic generalization of these facts in such a way that, corresponding to the trivial torsion theory we recover Rutter's result and for the Lambek torsion theory we get characterizations of the much larger class of left $\mathrm{QF}-3^{\prime}$ rings with ACC on left annihilators.

THEOREM 7. Let $\tau$ be a torsion theory of $R$-mod. The following conditions are equivalent:

(i) $R$ is $\tau$-artinian and $R / \mathrm{t}(R)$ has a minimal $\tau$-torsionfree cogenerator;

(ii) $R$ is $\tau$-artinian and every $\tau$-finitely generated submodule of $E(R / t(R))$ is $R / \mathbf{t}(R)$-torsionless;

(iii) $R$ is $\tau$-noetherian and each product of copies of $E(R / \mathbf{t}(R))$ is a projective $R / \mathbf{t}(R)$-module;

(iv) $R$ is $\tau$-noetherian and $E(R / \mathrm{t}(R))$ is projective as $R / \mathrm{t}(R)$-module.

PROOF. (i) $\Rightarrow$ (ii). If $R / \mathbf{t}(R)$ has a minimal $\tau$-torsionfree cogenerator, then it is clear that $E(R / \mathbf{t}(R))$ is $R / \mathrm{t}(R)$-torsionless from which (ii) follows.

(ii) $\Rightarrow$ (iii). Since $R$ is $\tau$-artinian, $R$ is $\tau$-noetherian by the Teply-Miller theorem. Let $E=E(R / \mathbf{t}(R))$. By [10, Corollary 3.3] we have that each product $E^{I}$ is a direct sum of injective envelopes of $\tau$-cocritical modules. Thus it is enough to prove that if $C$ is a $\tau$-cocritical submodule of $E^{I}$, then $E(C)$ is isomorphic to a submodule of $R / \mathrm{t}(R)$. If $C \leq E^{I}$, then there is a monomorphism $j: E(C) \rightarrow E^{I}$ 
and for some index $i$ of $I$ we have that $\left.\left(p_{i} \circ j\right)\right|_{C} \neq 0$ (where $p_{i}: E^{I} \rightarrow E$ is the $i$ th projection). Since $C$ is $\tau$-cocritical we see that $\left.\left(p_{i} \circ j\right)\right|_{C}$ is a monomorphism and, since $C$ is essential in $E(C)$, this implies that $p_{i} \circ j$ is a monomorphism, so that $E(C)$ is isomorphic to a submodule of $E$. Thus (ii) implies that every $\tau$-finitely generated submodule of $E(C)$ is $R / \mathrm{t}(R)$-torsionless and from Lemma 6 it follows that $E(C)$ is $\tau$-noetherian and hence $\tau$-finitely generated (see [7, Proposition 20.1]). Therefore, our hypothesis implies that $E(C)$ is $R / \mathrm{t}(R)$-torsionless and as before, we see that, in fact, $E(C)$ embeds in $R / \mathrm{t}(R)$.

(iii) $\Rightarrow$ (iv). This is obvious.

(iv) $\Rightarrow$ (i). Note that the proof of [9, Theorem 1] works to show that in our hypothesis $R$ is $\tau$-artinian. Moreover, $\tau$ is in this case strongly semiprime [7, Proposition 34.14] and hence, from Proposition 3, to show that $R / \mathrm{t}(R)$ has a minimal torsionfree cogenerator it will be enough to prove that $R / \mathbf{t}(R)$ has an injective cogenerator submodule which is $\tau$-finitely cogenerated. Since $R$ is $\tau$ artinian, ${ }_{R} R$ is $\tau$-finitely cogenerated [4, Proposition 1.5] and hence $E(R / t(R))$ is isomorphic to a finite direct sum of injective envelopes of $\tau$-cocritical modules. Let $\left\{E_{1}, \ldots, E_{n}\right\}$ be a representative set of the isomorphism classes of these injective envelopes. Since $E(R / \mathrm{t}(R))$ is $R / \mathrm{t}(R)$-torsionless by hypothesis, we see that each $E_{i}, i=1, \ldots, n$, is isomorphic to a submodule of $R / \mathbf{t}(R)$ and, as in the proof of Proposition 3, we have that $R / \mathrm{t}(R)$ contains a submodule which is isomorphic to $\bigoplus_{1}^{n} E_{i}$. It is clear that this submodule satisfies the required conditions and the proof is complete.

REMARK. If $\tau$ is the trivial torsion theory, then condition (i) of Theorem 7 is clearly equivalent to $R$ being a left artinian left QF-3 ring, so that the equivalence of (i) and (ii) gives [12, Corollary 3].

We may apply Theorem 7 to $\tau_{L}$ in order to characterize the rings which have a minimal $E\left({ }_{R} R\right)$-torsionless faithful left module and the DCC on left annihilators. Recall that a ring $R$ is called left 1-Gorenstein if $E\left({ }_{R} R\right)$ is projective [2] and that a module $X$ is $\Pi$-projective when each product of copies of $X$ is projective. We get

COROLLARY 8. The following conditions are equivalent for a ring $R$.

(i) $R$ has a minimal $E\left({ }_{R} R\right)$-torsionless faithful left module and satisfies the DCC on left annihilators.

(ii) $R$ is a left $\mathrm{QF}-3^{\prime}$ ring with DCC on left annihilators.

(iii) $R$ is a left 1-Gorenstein ring with ACC on left annihilators.

(iv) Every almost finitely generated submodule of $E\left({ }_{R} R\right)$ is torsionless and $R$ satisfies the DCC on left annihilators.

(v) Every almost finitely generated $E\left({ }_{R} R\right)$-torsionless module embeds in a free module and $E\left({ }_{R} R\right)$ is a fnite direct sum of injective envelopes of minimal rationally closed submodules. 
(vi) $E\left({ }_{R} R\right)$ is $\Pi$-projective.

(vii) Every $E\left({ }_{R} R\right)$-torsionless module embeds in a free module.

(viii) $R$ is a left QF-3' ring and every torsionless module embeds in a free module.

(ix) $R$ is a left QF-3' ring with semiprimary QF-3 maximal two-sided ring of quotients.

ProOF. Since in all cases any element of $E\left({ }_{R} R\right)$ is containd in a torsionless module, the ACC and the DCC on left annihilators are equivalent, respectively, to $R$ being $\tau_{L}$-noetherian and $\tau_{L}$-artinian. Now, the equivalence between (i), (ii), (iii) and (iv) follows in a strightforward way from Theroem 7 . From the equivalence between (iii) and (iv) of Theorem 7 it also follows that these conditions imply condition (vi).

(v) $\Leftrightarrow$ (vi). This follows from [8, Theorem 3.5] applied to the Lambek torsion theory.

That (vi) $\Rightarrow$ (vii) and (vii) $\Rightarrow$ (viii) are clear.

(viii) $\Rightarrow$ (iii). This follows from [10, Proposition 3.1].

That (iv) $\Rightarrow(\mathrm{ix})$ and (ix) $\Rightarrow$ (ii) are consequences of $[9$, Theorem 2].

REMARKS. Note that the equivalence between (iii) and (vii) above gives a proof of [9, Corollary 1] and shows that the converse of that corollary is also true. The class of rings which satisfy the conditions of Corollary 8 contains properly the class of left QF-3 rings with DCC on left annihilators (for example, ( $\left.\begin{array}{l}z \\ 0\end{array}\right)$ is left 1-Gorenstein and has ACC on left annihilators but is not left QF-3) and is contained properly in the class of rings which have a semiprimary QF-3 maximal two-sided ring of quotients (this class is characterized in [9, Theorem 2]). For instance, any commutative domain $R$ belongs to the latter class but $E\left({ }_{R} R\right)$ is not projective in the nontrivial cases (this also shows that in (iv) and (v) we cannot replace almost finitely generated modules by finitely generated ones). On the other hand, we also remark that it is not possible to replace DCC by ACC in conditions (i), (ii) and (iv) of Corollary 8. The example of Ringel and Tachikawa [11, page 60] of a left noetherian left QF-3 ring which is not right noetherian is not left 1-Gorenstein and hence does not satisfy the equivalent conditions of the above corollary.

We have obtained in Corollary 5 characterizations of the class of QF-3 rings with ACC on left annihilators. Other equivalent conditions are given in [13, Theorem] and in [9, page 385]. A different characterization is the following (recall that a ring $R$ is said to be left FC if $R$ is finitely cogenerated).

COROLLARY 9. The following conditions are equivalent for a ring $R$.

(i) $R$ is a QF-3 ring with ACC on left annihilators. 
(ii) $R$ satisfies the ACC on left annihilators and every almost finitely generated submodule of $E\left({ }_{R} R\right)$ and of $E\left(R_{R}\right)$ is torsionless.

(iii) $R$ is a left FC ring such that every almost finitely generated $E\left({ }_{R} R\right)$ torsionless left module embeds in a free module and every almost finitely generated $E\left(R_{R}\right)$-torsionless right module is torsionless.

(iv) $R$ is a left $\mathrm{QF}-3^{\prime}$ ring and $E\left(R_{R}\right)$ is $\Pi$-projective.

ProOF. The equivalences between (i) and (ii) and (i) and (iv) follow from Corollary 5 and Corollary 8.

(i) $\Rightarrow$ (iii). By Corollary $8,{ }_{R} R$ has an essential submodule of the form $\bigoplus_{1}^{n} C_{i}$, where the $C_{i}$ are minimal rationally closed left ideals (that is, $\tau_{L}$-saturated and $\tau_{L}$-cocritical left ideals). Since $R$ is right QF-3, $\tau_{L}$ is jansian and if $D$ is a minimal dense left ideal of $R$, each $D C_{i}$ is a simple left ideal of $R$ (see the proof of [7, Proposition 14.8]) which is essential in the uniform left ideal $C_{i}$ and hence ${ }_{R} R$ is finitely cogenerated. The rest of condition (iii) follows from Corollary 8 .

(iii) $\Rightarrow$ (ii). Clearly (iii) implies that $R$ satisfies condition (v) of Corollary 8 , so that $R$ has ACC on left annihilators and hence we get that (ii) holds.

For left nonsingular rings, the $\tau_{L}$-dense left ideals are precisely the essential left ideals of $R$ [15]. Denoting by $Q=R_{\tau_{L}}$ the maximal left ring of quotients of $R$, we get

PROPOSITION 10. The following conditions are equivalent for a ring $R$.

(i) $R$ is a let nonsingular left $\mathrm{QF}-3^{\prime}$ ring with $\mathrm{ACC}$ (or DCC) on left annihilators.

(ii) $R$ is a left nonsingular left finite-dimensional left $\mathrm{QF}-3^{\prime}$ ring.

(iii) $Q$ is semisimple and ${ }_{R} Q$ is projective.

(iv) $Q$ is semisimple and every nonsingular left $R$-module embeds in a free module.

(v) $R$ is a left $\mathrm{QF}-3^{\prime}$ ring with semisimple maximal two-sided ring of quotients.

(vi) $R$ is a right FC ring with projective right socle and every finitely generated nonsingular right $R$-module embeds in a free module.

Proof. (i) $\Leftrightarrow$ (ii). We observed in the proof of Corollary 5 that if $R$ is left QF-3', then ACC (or DCC) on left annihilators is equivalent to $R$ being $\tau_{L^{-}}$ noetherian. Thus the equivalence between (i) and (ii) follows from [15, Proposition XIII.3.2 and Proposition XIII.3.3].

(ii) $\Leftrightarrow$ (iii) $\Leftrightarrow$ (iv). These equivalences follows readily from [2, Proposition 2.1 and Theorem 2.5], since $Q$ is semisimple if and only if $R$ is left nonsingular and left finite-dimensional. 
(iv) $\Rightarrow$ (v). Since, by (iv), $R$ is left nonsingular and finite-dimensional it follows from [15, Corollary XII.7.3], that $Q$ is also the maximal right ring of quotients of $R$.

$(\mathrm{v}) \Rightarrow(\mathrm{vi})$. By (v), $R$ is right nonsingular and right finite-dimensional so that, by [15, Corollary XII.7.3] every finitely generated nonsingular right $R$-module embeds in a free module. Furthermore, from Corollary 8 it follows that ${ }_{R} Q$ is projective and so $R$ is a right FC ring with projective right socle by [14, Corollary 2.5].

(vi) $\Rightarrow$ (iii). If $Q^{\prime}$ denotes the maximal right ring of quotients of $R$, then $Q^{\prime}$ is semisimple and ${ }_{R} Q^{\prime}$ is projective by [14, Corollary 2.5]. From [15, Corollary XII.7.3] it follows that $Q=Q^{\prime}$, which completes the proof.

REMARKS. The left nonsingular left $\mathrm{QF}-3^{\prime}$ rings which contain no infinite sets of orthogonal idempotents are characterized in [2, Theorem 2.5]. The class of rings defined by Proposition 10 is smaller than the class of rings such that $Q$ is semisimple and every finitely generated nonsingular left $R$-module embeds in a free module, for among the rings satisfying the last conditions are all the two-sided orders in semisimple rings [15, Proposition II.4.6]. Also, there are rings $R$ with maximal right ring of quotients $Q^{\prime}$ semisimple and ${ }_{R} Q^{\prime}$ projective (these rings are studied in [14, Corollary 2.5]) which do not satisfy the conditions of Corollary 11. An example is given by the ring considered in [15, Exercise XII.5]. On the other hand, $\left(\begin{array}{l}z \\ 0\end{array}\right)$ satisfies the conditions of Proposition 10 but not their left-right symmetric, for it is not a right QF-3' ring. On the contrary, if we replace left QF-3' by left QF-3 in (i), (ii) or (v), we get a class of rings which is left-right symmetric [2, Theorem 2.8].

\section{References}

[1] F. W. Anderson and K. R. Fuller, Rings and categories of modules, Graduate Texts in Math., 13 (Springer-Verlag, New York, 1974).

[2] G. Baccella, 'The structure of QF-3 rings with zero singular ideal', Comm. Algebra 15 (1987), 1393-1446.

[3] R. R. Colby and E. A. Rutter, Jr., 'QF-3 rings with zero singular ideal', Pacific J. Math. 28 (1969), 303-308.

[4] J. L. García Hernández and J. L. Gómez Pardo, ' $V$-rings relative to Gabriel topologies', Comm. Algebra 13 (1985), 59-83.

[5] J. L. García Hernández and J. L. Gómez Pardo, 'Self-injective and PF endomorphism rings', Israel J. Math. 58 (1987), 324-350.

[6] J. L. García Hernández and J. L. Gómez Pardo, 'Closed submodules of free modules over the endomorphism ring of a quasi-injective module', Comm. Algebra 16 (1988), 115-137.

[7] J. S. Golan, Torsion theories, Longman, Harlow, 1986.

[8] J. L. Gomez Pardo, 'Embedding cyclic and torsion-free modules in free modules', Arch. Math. 44 (1985), 503-510. 
[9] K. Masaike, 'Semiprimary QF-3 quotient rings', Comm. Algebra 11 (1983), 377-389.

[10] R. W. Miller and M. L. Teply, 'The descending chain condition relative to a torsion theory', Pacific J. Math. 83 (1979), 207-218.

[11] C. M. Ringel and H. Tachikawa, 'QF-3 rings', J. Reine Angew. Math. 272 (1975), 49-72.

[12] E. A. Rutter, Jr., 'A characterization of QF-3 rings', Pacific J. Math. 51 (1974), 533-536.

[13] E. A. Rutter, Jr., 'QF-3 rings with ascending chain condition on annihilators', $J$. Reine Angew. Math. 277 (1975), 40-44.

[14] E. A. Rutter, Jr., 'Dominant modules and finite localizations', Tôhoku Math. J. 27 (1975), 225-239.

[15] B. Stenström, Rings of quotients (Springer-Verlag, Berlin, Heidelberg, New York, 1975).

[16] H. Tachikawa, 'On left QF-3 rings', Pacific J. Math. 32 (1970), 255-268.

Departamento de Matemáticas

Universidad de Murcia

30001 Murcia,

Spain 